\title{
Taller de cuentos madre naturaleza para mejorar las actitudes ambientales de estudiantes de primaria, Luya 2018
}

\section{Story workshop mother nature to improve the environmental attitudes of primary students, Luya 2018}

Kelly Rocio Rivera Sopla ${ }^{1}$ Ivon Perpetua Hidalgo Velaysosa ${ }^{2}$

\section{RESUMEN}

El estudio de tipo cuantitativo-cualitativo, con pre test-post test y grupo control, tuvo como propósito determinar la influencia del taller cuentos "Madre naturaleza" en la mejora de actitudes ambientales. Se trabajó con 20 estudiantes de nivel primaria durante dos meses. Las actividades estuvieron relacionadas con el tratamiento de los residuos sólidos, el uso adecuado de pilas y la conservación del mono choro cola amarilla. Los resultados demuestran que hubo influencia significativa en el cambio de actitudes ambientales cognitivas (de 6,65 puntos en el pre test a 17,5 puntos en el post test con un valor de la prueba $Z=0,435$ en el pre test y 0,074 en el post test), actitudes ambientales afectivas (de 20,60 puntos en el pre test a 28,60 puntos en el post test con un valor de la prueba $Z=0,080$ en el pre test y 0,008 en el post test), y actitudes ambientales conductuales reflejándose en el desarrollo de 27 actividades colectivas, tanto en la escuela y fuera de ella. El aporte principal del trabajo es haber logrado alta participación comprometida de los estudiantes en acciones de mitigación, así como haber incrementado sus conocimientos y valoración por los recursos naturales y el consecuente cambio de actitudes respecto a la problemática ambiental.

Palabras clave: Actitud ambiental, taller de cuentos ambientales.

\begin{abstract}
The study of quantitative type-qualitative, with pre-test-post test and control group, was intended to determine the influence of the workshop tales "Mother nature" in the improvement of environmental attitudes. We worked with 20 primary-level students for two months. The activities were related to the treatment of solid waste, the proper use of batteries and the conservation of the yellow-tailed Woolly monkey. The results show that there was significant influence on the change of cognitive environmental attitudes (from 6.65 points in the pre-test to 17.5 points in the post-test with a value of the test $Z=0,435$ In the pre-test and 0.074 in the post-test ), affective environmental attitudes (from 20.60 points in the pre-test to 28.60 points in the post-test with a value of the test $Z=0.080$ in the pre-test and 0.008 in the post-test), and behavioural environmental attitudes reflecting in the development of 27 collective activities, both at school and outside. The main contribution of the work is to have achieved a high commitment of the students in mitigation actions, as well as having increased their knowledge and valuation of natural resources and the consequent change of attitudes regarding environmental problems.
\end{abstract}

Keywords: Environmental attitude, environmental story workshop.

${ }^{1}$ Universidad Nacional Toribio Rodríguez de Mendoza de Amazonas. Email: kellyrocio-3@hotmail.com

${ }^{2}$ Universidad Nacional Toribio Rodríguez de Mendoza de Amazonas. Email: michikitas@hotmail.com 


\section{INTRODUCCIÓN}

La naturaleza y la sociedad actual enfrentan una serie de problemas, que requieren ser tratados de manera global, integral y urgente. En la interrelación dialéctica entre la naturaleza y la sociedad surgen los problemas ambientales.

En la actualidad los factores que afectan nuestro planeta son: el mal procesamiento de los deshechos residuos sólidos - basura, químicos como amoniaco, contaminación del aire, agua, suelo, flora y fauna, la deforestación - tala de árboles, destrucción de habitad - ecosistema, impacto atmosférico - efecto invernadero, calentamiento global - cambio climático y el uso irracional de los recursos naturales.

Ante esta situación, y en función de las necesidades, intereses y problemas de la sociedad y frente a la situación ambiental actual han formulado muchas políticas de recuperación del medio ambiente y mejora de calidad de vida en distintas esferas a nivel global, nacional, regional y local.

En la Institución Educativa "José Antonio Encinas Franco" - Luya, se ha podido evidenciar que se necesita reforzar en los niños el cuidado de su medio. Por otro lado, se observó en los tachos no solo basura "común" sino pilas en estado de descomposición, razón por lo cual se realizó una encuesta con fines de diagnóstico a los niños del cuarto grado, encontrando que el 100\% de los niños afirmaron tener objetos con pilas en sus casas; el $13.04 \%$ afirmaron tener radio a pilas en su hogar, el $30.44 \%$ tienen juguetes que funcionan a base de pila, el $4.35 \%$ afirma tener celular, el $52.17 \%$ dicen tener todos los objetos que requiere de las pilas para que puedan funcionar; por otro lado, la misma encuesta revela que el $4.35 \%$ de los niños después de utilizar las pilas lo tiran a la huerta; el $73.91 \%$ aduce poner la pila junto a la basura y el $21.74 \%$ dicen enterrarlo en sus patios, situación que evidenció que la pila lo perciban como una basura común y corriente ignorando que las pilas y baterías son de alto riesgo para la salud de las personas y de todos los seres vivos que habitan en la naturaleza, siendo así una causa principal para la contaminación del medio ambiente y deterioro de la flora y fauna silvestre.

En esta misma dirección se indagó también a los estudiantes sobre animales en peligro de extinción en nuestra región Amazonas, sin embargo, solo un $4,35 \%$, conocen por ejemplo, que el mono choro cola amarilla se encuentra en peligro de extinción y el $95.65 \%$ lo desconocían.

En este contexto, la educación ambiental constituye una herramienta para trabajar y, poco a poco cambiar las actitudes ambientales de los estudiantes; ya que el propósito fundamental es desarrollar y fortalecer una conciencia que propicie y estimule el respeto y la convivencia de las personas hacia su medio ambiente natural y cultural; en el marco de un conocimiento apropiado de los recursos y la capacidad de uso sostenible.

Tal como lo señala Yoplac (2016) "los programas de educación ambiental deben partir de la realidad objetiva, involucrar a la población organizada" (p.41). El objeto del presente estudio fue determinar la influencia del taller de cuentos "Madre naturaleza" en la mejora de actitudes ambientales cognitivas, afectivas y conductuales de los estudiantes, relacionados con el tratamiento de los residuos sólidos, uso de las pilas y cuidado del mono choro cola amarilla, problemas relacionados con la vida misma del estudiante.

Anguiano (2003) manifiesta que hablar de educación ambiental significa hablar de conocimientos, aptitudes, actitudes, valores y acciones, de todos estos los valores juegan un importante papel, ya que a través de estos los conocimientos y aptitudes pueden transformarse en actitudes y acciones, elementos claves en la Educación Ambiental, los ámbitos donde los adquirimos son principalmente la escuela, la familia y la sociedad.

Hay muchas formas de hacer educación ambiental, particularmente en el presente trabajo se ha tomado como base los cuentos ambientales, específicamente tres cuentos del libro "Madre naturaleza 3" (Yoplac, 2015): el monstruo tragabasuras, la muerte del arbolito y las lágrimas de tristeza del monito bonito; Yoplac (2014), plantea cuatro principios generales de un cuento ambiental: i) los cuentos son una herramienta educativa esencialmente colectiva, ii) el contenido del cuento está relacionado con un problema ambiental, iii) la historia del cuento encierra una verdad científica o se sustenta en la sabiduría popular, iv) el lenguaje utilizado en la construcción de los cuentos es sencillo y propio de los pobladores en contexto de la investigación.

En este contexto es que la presente investigación se orienta a trabajar con estudiantes de primaria de una institución educativa rural en la búsqueda de mejorar sus actitudes ambientales y convertirse en agentes protagonistas de conocimiento, valoración y transformación de la realidad en bien del cuidado natural y social.

\section{MATERIAL Y MÉTODOS}

\section{Diseño de la investigación}

La presente investigación es de tipo cuasi experimental, con grupo control no aleatorio. 


\section{Población, muestra y muestreo}

La población estuvo representada por 244 estudiantes entre niños y niñas de la Institución Educativa No 18132 "José Antonio Encinas Franco", de Luya, región Amazonas.

La muestra estuvo conformad por 20 estudiantes del cuarto grado.

El muestreo fue no probabilístico intencional, ya que las investigadoras elegimos la muestra según las características del contexto del desarrollo de la investigación.

\section{Fuentes de información}

Se utilizaron diversas fuentes como: fichas de observación grupal, evaluaciones pre test y post test, investigaciones de otros autores, libros relacionados con el tema de investigación, autoridades, Institución Educativa y otros.

\section{Métodos}

En la investigación se utilizó el método científico como método general. Como método específico se utilizó el experimental, analítico-sintético.

\section{Instrumentos de investigación}

Los instrumentos que se utilizaron en la presente investigación fueron: el test, escala de actitudes ambientales y las guías de observación.

Para la validez de nuestros instrumentos se ha determinado mediante la opinión de tres expertos en donde el resultado fue de 0,080 puntos y para evaluar su fiabilidad se aplicó la prueba de alfa de Cronbach con un puntaje de 0,724.Descripción del Proceso de Trabajo

Fase 1. Se hizo un diagnóstico de la problemática ambiental en la Institución Educativa № 18132 José Antonio Encinas Franco - de Luya, 2018, especialmente con los estudiantes del cuarto grado de primaria. Previamente se aplicó el pre test a ambos grupos.

Fase 2. Se elaboró el taller de cuentos "Madre naturaleza" que incluye 27 sesiones de aprendizaje.

Fase 3. Se presentó y aplicó el taller durante dos meses desde las propias aulas y fuera de ella. Finalizado el taller se aplicó el pos test a ambos grupos.

\section{Procesamiento y presentación de los datos}

Luego del suministro de los instrumentos de investigación, se organizó, presentó y procesó los datos en tablas y figuras antes y después de aplicar el taller de cuentos "Madre naturaleza" en el grupo experimental. Luego, se probó y contrastó la hipótesis de la investigación con el estadístico $t$ de student, de acuerdo al diseño de la investigación, al cálculo de la T- calculada y la T- tabulada sobre la mejora de las actitudes ambientales. Así mismo, para el análisis de los datos se utilizó tanto la estadística descriptiva como la estadística inferencial.

Finalmente, acorde con el diseño de la investigación, los análisis estadísticos se realizaron con el programa computacional SPSS (Statistical Package for Social Sciencies) en su última versión (versión 25.0). Asimismo, utilizamos para la sistematización de nuestros datos el paquete de Microsoft Office, especialmente, Microsoft Excel versión 2010, que es un programa integrado que combina en un solo paquete una hoja de cálculo, gráficos y macros, bajo el sistema operativo Windows.

\section{Descripción de la prueba de hipótesis}

Para evaluar el nivel de significancia del taller de cuentos "Madre naturaleza", en la mejora de actitudes ambientales cognitivas se aplicó el parámetro estadístico $\mathrm{t}$ de student con una probabilidad del 95\% y un error de significación de $5 \%$ cuyo p-valor $=0.000$, encontrándose que la prueba fue significativa.

Del mismo modo, se utilizó la prueba de normalidad Shapiro- Wilk para el nivel de conocimiento con una significancia en el pre test de 0.435 y 0,074 en el post test y para medir el nivel valorativo se utilizó la prueba de Wilcoxon.

\section{RESULTADOS}

Los resultados se presentan tomando en cuenta las actitudes cognitivas, valorativas y conductuales. Para las dos primeras se presentan tablas que compraran los resultados del pre test y post test del grupo experimental, y para las actitudes conductuales se mencionan las actividades colectivas realizadas cooperativamente.

Resultados sobre actitudes ambientales cognitivas del grupo experimental y control

Tabla 1. Puntajes medios sobre el conocimiento de residuos sólidos, pilas y mono choro cola amarilla.

\begin{tabular}{l|r|r|r}
\hline & \multicolumn{1}{|c|}{ Media } & \multicolumn{1}{c|}{ Varianza } & \multicolumn{1}{c}{$\begin{array}{c}\text { Desv. } \\
\text { Estandar }\end{array}$} \\
\hline Pre test & 6.65 & 5.924 & 2.43 \\
\hline Post test & 17.5 & 2.263 & 1.5 \\
\hline
\end{tabular}

Nota: La escala de evaluación de la prueba es vigesimal.

En la tabla del grupo experimental se evidencia que el puntaje medio en el pre test fue de $6,65 \mathrm{y}$ de 17,5 en el 
post test, apreciándose un incremento antes y después de la aplicación del taller.

\begin{tabular}{l|c|c|c|c}
\hline & Media & Varianza & $\begin{array}{l}\text { Desv. } \\
\text { Estándar }\end{array}$ & $\begin{array}{l}\text { Media } \\
\text { na }\end{array}$ \\
\hline $\begin{array}{l}\text { Puntaje } \\
\text { P. }\end{array}$ & 7.5 & 4.68 & 2.16 & 8 \\
Entrada & 9.9 & 1.88 & 1.37 & 10 \\
\hline $\begin{array}{l}\text { Puntaje } \\
\text { P. Salida }\end{array}$ & 9.9 \\
\hline
\end{tabular}

Nota: La escala de evaluación de la prueba es vigesimal

El puntaje promedio de los estudiantes del grupo control en la prueba de entrada es de 7.5 y en la prueba de salida es de 9.9. Los puntajes de la prueba de salida tienen menos variabilidad a comparación con los puntajes de la prueba de entrada.

Tabla 2. Distribución porcentual del nivel de conocimiento sobre residuos sólidos, pilas y mono cola amarilla

\begin{tabular}{l|c|c|c|c}
\hline \multirow{2}{*}{} & \multicolumn{2}{|c|}{ Pre Test } & \multicolumn{2}{c}{ Post Test } \\
\cline { 2 - 5 } & f & $\%$ & f & \% \\
\hline Nivel bajo & 12 & 60 & 0 & 0 \\
\hline Nivel medio & 8 & 40 & 0 & 0 \\
\hline Nivel alto & 0 & 0 & 20 & 100 \\
\hline Total & $\mathbf{2 0}$ & $\mathbf{1 0 0}$ & $\mathbf{2 0}$ & $\mathbf{1 0 0}$
\end{tabular}

Nota: nivel bajo $=1-7$ puntos; nivel medio $=8-14$ puntos; nivel alto $=15-20$ puntos.

En la tabla, del grupo experimental se observa que el pre test el nivel bajo es el más predominante, con un $60 \%$, comparándolo con el nivel medio, que tiene un $40 \%$. No obstante, en el post test el nivel predominante al $100 \%$ es alto.

\begin{tabular}{l|c|c|c|c}
\hline \multirow{2}{*}{} & \multicolumn{2}{|c|}{ Pre test } & \multicolumn{2}{c}{ Post test } \\
\cline { 2 - 5 } & $\mathrm{f}$ & $\%$ & $\mathrm{f}$ & $\%$ \\
\hline Nivel bajo & 9 & 45 & 1 & 5 \\
\hline $\begin{array}{l}\text { Nivel } \\
\text { medio }\end{array}$ & 11 & 55 & 19 & 95 \\
\hline Total & $\mathbf{2 0}$ & $\mathbf{1 0 0}$ & $\mathbf{2 0}$ & $\mathbf{1 0 0}$ \\
\hline
\end{tabular}

Nota: nivel bajo $=1-7$ puntos; nivel medio $=8-14$ puntos; nivel alto $=15-20$ puntos.

En la tabla del grupo control se aprecia que el nivel medio es el más predominante, con un $55 \%$, seguido del nivel bajo, con un $45 \%$. En la segunda prueba, el nivel medio es el que más predomina, con un $95 \%$, frente al 5\% del nivel bajo.

Resultados sobre actitudes ambientales afectivas del grupo experimental y control

Tabla3. Distribución porcentual del nivel de predisposición de realizar actividades mitigantes sobre residuos sólidos, uso correcto de pilas y preservación del mono choro cola amarilla.

\begin{tabular}{l|c|c|c|c}
\hline \multirow{2}{*}{} & \multicolumn{2}{|c|}{ Pre Test } & \multicolumn{2}{c}{ Post Test } \\
\cline { 2 - 5 } & f & $\mathbf{\%}$ & f & $\%$ \\
\hline Nivel bajo & 0 & 0 & 0 & 0 \\
\hline Nivel medio & 10 & 50 & 0 & 0 \\
\hline Nivel alto & 10 & 50 & 20 & 100 \\
\hline Total & $\mathbf{2 0}$ & $\mathbf{1 0 0}$ & $\mathbf{2 0}$ & $\mathbf{1 0 0}$ \\
\hline
\end{tabular}

Nota: nivel bajo $=$ en desacuerdo; nivel medio = indiferente; nivel alto $=$ de acuerdo.

En la tabla, se aprecia que en el pre test, tanto el nivel medio y nivel alto tienen el mismo porcentaje, ambos con un 50\%; sin embargo, en el post test, se puede apreciar el incremento del porcentaje del nivel alto, que ahora tiene un $100 \%$ de predisposición en realizar acciones de mitigación ambiental.

\begin{tabular}{l|c|c|c|c}
\hline \multirow{2}{*}{} & \multicolumn{2}{|c|}{ Pre test } & \multicolumn{2}{c}{ Post test } \\
\cline { 2 - 5 } & $\mathrm{f}$ & $\%$ & $\mathrm{f}$ & $\%$ \\
\hline $\begin{array}{l}\text { Nivel } \\
\text { medio }\end{array}$ & 5 & 25 & 7 & 35 \\
\hline Nivel alto & 15 & 75 & 13 & 65 \\
\hline Total & $\mathbf{2 0}$ & $\mathbf{1 0 0}$ & $\mathbf{2 0}$ & $\mathbf{1 0 0}$ \\
\hline
\end{tabular}

Nota: nivel bajo = en desacuerdo; nivel medio = indiferente; nivel alto $=$ de acuerdo.

Se aprecia que después de la primera y segunda evaluación a los estudiantes del grupo control en el Nivel de Valoración, el nivel alto es el predominante con $75 \%$, frente al nivel medio con $25 \%$, en la primera prueba. Se puede notar un pequeño incremento en el nivel medio, pasando de $25 \%$ a un $35 \%$ y en la segunda prueba, la disminución del nivel alto, de $75 \%$ a $65 \%$.

\section{Resultados sobre actitudes ambientales conductuales}

Se realizó un total de 27 actividades académicasde las cuales 10 estuvieron relacionados con el tratamiento de residuos sólidos, 8 con el uso de pilas y 9 relacionadas con conservación del mono choro cola amarilla, desarrolladas teniendo en cuenta los eventos de sensibilización, concientización y acción, realizadas con los estudiantes y en algunos casos con los padres de familia y otros actores.

En el manejo de residuos sólidos se trabajó con el cuento "el monstro traga basuras" las siguientes actividades: narración; lectura; dibujando el 
monstruo traga basura de Tincas; escenificación; explicación; dando un final feliz al cuento; elaboración de tachos de basura para el aula y contenedores de botellas descartables; clasificación de los tachos de basura: orgánico (color verde), inorgánico reciclable (color marrón) e inorgánico no reciclable (color rojo); visita al botadero de basura de Tincas y elaboración de la compostera.

En el uso de pilas se trabajó se trabajó con el cuento "la muerte del arbolito" las siguientes actividades: narración; lectura; dibujando sobre el peligro de las pilas; escenificación; explicación; dando un final feliz al cuento; concurso de recojo de pilas y elaboración del ataúd para el depósito de las pilas.

Para la conservación del mono choro cola amarilla se trabajó se trabajó con el cuento "las lágrimas del monito bonito" las siguientes actividades: narración; lectura; recreando los dibujos; escenificación; explicación; dando un final feliz al cuento, teatralización; conferencia de Alma Hernández Jaramillo (Bióloga) sobre el peligro de extensión del mono choro cola amarilla y concurso: de pintura: el monito en peligro de extinción, de expresión oral: exposición de los dibujos de cada cuento elaborados en el taller y de producción de texto: final feliz a cada cuento.

\section{DISCUSIÓN}

El taller de cuentos "Madre Naturaleza", permitió mejorar el cambio de actitudes, cognitiva, valorativas y conductuales con los estudiantes del grupo experimental tal como lo demuestra nuestros resultados en el nivel cognitivo en pre test con un puntaje en el nivel bajo $60 \%$, en el nivel medio de $40 \%$ y en el pos test con un $100 \%$ en el nivel alto, hecho que nos permite comparar con los resultados de Panduro(2013) sobre el nivel de conocimiento en la reducción de la contaminación ambiental en las juntas vecinales, con un puntaje en el pre test de $8 \%$ en el nivel bueno, en el nivel regular $22 \%$ y en nivel malo el $70 \%$ y en el pos test con un $76 \%$ en el puntaje bueno, $16 \%$ en el puntaje regular y $8 \%$ en el nivel malo siendo sus resultados menores a los nuestros.

El uso de cuentos en la educación ambiental posibilita la participación del estudiante en el conocimiento, valoración y acción de la problemática ambiental, tanto de la escuela como de la comunidad.

Evidentemente los resultados muestran que el taller de cuentos "Madre naturaleza" (variable independiente) ha tenido un influencia positiva en los estudiantes de la Institución Educativa "José Antonio Encinas Franco" - Luya, para la mejora de actitudes ambientales cognitivas (de 6,65 puntos en el pre test a
17,5 puntos en el post test con un valor de la prueba $Z$ $=0,435$ en el pre test y 0,074 en el post test) logrando que el $100 \%$ de los estudiantes conozcan sobre el cuidado de nuestro medio ambiente.

A través de la investigación con el uso del taller de cuentos "Madre Naturaleza", coincidimos con Ramos (2015), donde sostienen que "el Programa mis cuentos ecológicos produjo efectos positivos y significativos en el desarrollo de las actitudes ambientales en los niños...". Tal como se evidencia en nuestros resultados obtenidos, con estudiantes del nivel escolar, demostramos que los cuentos sirven como posibles soluciones a los graves problemas por falta de conocimiento, valoración y acciones que permitan el cambio de actitudes ambientales, haciendo de ello un hábito en los estudiantes del cuarto grado de la institución educativa "José Antonio encinas Franco" - Luya, en el nivel cognitivo, valorativo y conductual. Además, hubo influencia significativa en el cambio de actitudes ambientales afectivas (de 20,60 puntos en el pre test a 28,60 puntos en el post test con un valor de la prueba $Z=0,080$ en el pre test y 0,008 en el post test).

También hacemos evidencia que en los resultados de las actitudes ambientales afectivas en el grupo experimental los resultados que obtuvimos en el pre test en el nivel medio 50\% y en el nivel bajo 50\% logrando alcanzar el 100\% en el post test después de aplicar el taller de cuentos "Madre Naturaleza" de esta manera con paramos con los resultados de Yoplac (2013) en sus resultados de nivel valorativo con su programa de cuantos ecológicos obteniendo un puntaje 0,3totalmente de acuerdo, 42.9\%, indiferente $39,0 \%$, en desacuerdo $16,9 \%$ y totalmente en desacuerdo 1,0 puntajes obtenidos en el pre test y en el post test con un $49.4 \%$ en totalmente de acuerdo, $50,6 \%$ en acuerdo, evidenciándose así que un taller de cuentos tiene más aceptación en estudiantes de nivel escolar que en personas adultas.

Nuestra preocupación fue cuando percibimos que en la institución educativa “José Antonio Encinas Franco" - Luya, los estudiantes conocen ciertos temas como el reciclaje pero desconocían en su totalidad el mal uso y lo dañino que puede ser para la salud de los seres vivos arrojar la basura en cualquier lugar, no solo eso sino también el mal uso de productos químicos como las pilas y peligro de extinción de los animales, en tal sentido el haber trabajado con los estudiantes a través del taller de cuentos "Madre Naturaleza" se pudo lograr la toma de conciencia y mejora de nuestra calidad de vida y de los animales de nuestro entorno tal como señala Moreno, Corraliza, y Ruiz (2005) determinaron que la población encuestada conoce de manera general 
los problemas ambientales sin embargo existe mayor información en los temas de ruido, espacios naturales, transporte y reciclaje y menos en basura y agua quedando en posiciones intermedias la contaminación global, los productos químicos, la energía y la biodiversidad.

El aporte principal de la investigación fue demostrar que el taller de cuentos "Madre Naturaleza", contribuye favorable y significativamente en el mejoramiento de cambio de actitudes ambientales, en el nivel de conocimientos, mayor valoración y mejor capacidad de acción para la protección de los animales en peligro de extinción, manejo de los recursos sólidos y el uso adecuado de las pilas. De la misma forma, la ejecución de la investigación ha permitido conocer que el taller de cuentos es una herramienta unificadora entra la teoría y la práctica, y socializadora entre las investigadoras, estudiantes y población en su conjunto, y promueve la identificación, la mitigación de los problemas ambientales, el respeto y cuidado a los seres vivos tal como lo demuestran los Autores citados en nuestro trabajo de investigación.

\section{CONCLUSIONES}

El taller de cuentos "Madre naturaleza" tiene una influencia positiva en la mejora de actitudes ambientales cognitivas, afectiva y conductuales de los estudiantes relacionados con el tratamiento de los residuos sólidos, uso de las pilas y cuidado del Mono Choro Cola Amarilla.

Se ha determinado que el taller de cuentos "Madre naturaleza mejora significativamente las actitudes ambientales cognitivas de los estudiantes de la Institución Educativa "José Antonio Encinas Franco" Luya, evidenciándose en que las medias de los puntajes obtenidos sobre el nivel de conocimientos fueron de 6,65 en el pre test y de 17,5 en el post test.

Se ha determinado que el taller de cuentos Madre naturaleza mejora significativamente las actitudes ambientales valorativas de los estudiantes de la Institución Educativa “José Antonio Encinas Franco" Luya, evidenciándose en el incremento de predisposición en realizar acciones de mitigación ambiental que pasa del $50 \%$ en el pre test al $100 \%$ en el post test.

El taller de cuentos |"Madre naturaleza mejora significativamente las actitudes ambientales conductuales de los estudiantes de la Institución Educativa "José Antonio Encinas Franco" Luya, evidenciándose en el desarrollo de 27 actividades encaminadas a la mitigación de problemas ambientales relacionados con el tratamiento de los residuos sólidos, uso de las pilas y cuidado del mono choro cola amarillo.

\section{REFERENCIAS BIBLIOGRÁFICAS}

Anguiano, M. (2003). Proyecto Ecológico en educación primaria Mi Escuela Ecológica. México: Colima.

Panduro, H. (2013). Programa de educación a mbiental para reducir la contaminación en las juntas vecinales de la ciudad de Chachapoyas. (Tesis para optar el grado académico de doctora en ciencias ambientales. Universidad Nacional de Trujillo). $\mathrm{R}$ e c u p e r a d o d e : http://dspace.unitru.edu.pe/bitstream/h andle/unitru/4992/tesis\%20doctoral $\% 2$ 0hilda $\% 20$ panduro $\% 20$ baz $\%$ c3\%81n.p df? sequence $=1 \&$ isallowed $=y$

Ramos, Á. J. (2015). Programa mis cuentos ecológicos para desarrollar actitudes ambientales en los niños del 3er grado de la institución educativa $N^{\circ} 32008$, Señor de los Milagros - Huánuco 2014, para obtener el título de licenciado en Educación en Educación básica: Inicial y Primaria. Universidad de Huánuco. Perú.

Yoplac, M. (Julio - Diciembre, 2016). Programa de cuentos para la mejora de actitudes ambientales de los pobladores de Alizo, Amazonas. UNTRM, Ciencias Sociales y Humanidades, 1(1), 29-36.

Yoplac, M. (2015). Madre naturaleza, cuentos para crear conciencia ambiental 3. Perú: EDEPE

Yoplac, M. (2014). Los cuentos y la educación ambiental. Revista Ecos del pueblo. 5 (4) 64-84. Chachapoyas.

Yoplac, M., Guevara, R. y Fernández, J., (2009). Los cuentos como instrumentos en la formación de conciencia ambiental de los estudiantes de $4^{\circ}, 5^{\circ}$ y $6^{\circ}$ grados de educación primaria rural del Valle de Guayabamba, provincia Rodríguez de Mendoza, región Amazonas. (Tesis de posgrado, Universidad Nacional Toribio Rodríguez de Mendoza de Amazonas, Chachapoyas). 